THE ASTROPHYSICAL JOURNAL, 517:719-724, 1999 June 1

\title{
OPTIMAL MICROLENSING OBSERVATIONS
}

\author{
ANDREW GOULD ${ }^{1}$ \\ Ohio State University, Department of Astronomy, 174 West 18th Ave., Columbus, OH 43210; gould@astronomy.ohio-state.edu \\ Received 1998 August 3; accepted 1999 January 10
}

\begin{abstract}
One of the major limitations of microlensing observations toward the Large Magellanic Cloud (LMC) is the low rate of event detection. What can be done to improve this rate? Is it better to invest telescope time in more frequent observations of the inner high-surface-brightness fields or in covering new, less populated outer fields? How would a factor 2 improvement in CCD sensitivity affect the detection efficiency? Would a series of major (factor 2-4) upgrades in telescope aperture, seeing, sky brightness, camera size, and detector efficiency increase the event rate by a huge factor, or only marginally? I develop a simplified framework to address these questions. With observational resources fixed at the level of the MACHO and EROS experiments, the biggest improvement (factor $\sim 2$ ) would come by reducing the time spent on the inner $\sim 25 \mathrm{deg}^{2}$ and applying it to the outer $\sim 100 \mathrm{deg}^{2}$. By combining this change with the characteristics of a good medium-size telescope $\left(2.5 \mathrm{~m}\right.$ mirror, $1^{\prime \prime}$ point-spread function, thinned CCD chips, $1 \mathrm{deg}^{2}$ camera, and dark sky), it should be possible to increase the detection of LMC events to more than $100 \mathrm{yr}^{-1}$ (assuming current estimates of the optical depth apply to the entire LMC).

Subject headings: dark matter — Galaxy: halo — gravitational lensing - Magellanic Clouds
\end{abstract}

\section{INTRODUCTION}

Microlensing observations toward the Large Magellanic Cloud (LMC) have yielded puzzling results: the event rate toward the LMC is much too high to be caused by known populations of stars, but the $M \sim 0.4 M_{\odot}$ mass of the lenses (as inferred from the $t_{\mathrm{E}} \sim 40$ day timescale of the events) is too heavy to be because of a halo of brown dwarfs (Alcock et al. 1997a; Ansari et al. 1997). Moreover, if the LMC events were due to halo lenses, one would expect similar events toward the Small Magellanic Cloud (SMC). However, both of the events detected to date toward the SMC show signs of being SMC self-lensing (Palanque-Delabrouille et al. 1998; Afonso et al. 1998; Alcock et al. 1997b, 1999; Albrow et al. 1999; Udalski et al. 1998; Rhie et al. 1999).

The two most difficult obstacles to unraveling the nature of the lenses are the low overall event detection rate and the lack of information about individual events. The first 2 years of LMC observations by the MACHO collaboration yielded only eight candidate events over the inner $11 \mathrm{deg}^{2}$ (Alcock et al. 1997a), making it difficult to discern nonuniformities in the spatial distribution of the events, which one would expect if they were predominantly due to LMC selflensing. For most events, the only information is the timescale $t_{\mathrm{E}}$, which is a complicated combination of the three quantities one would like to know about the lens, its mass $M$, distance $d_{\mathrm{ol}}$, and transverse speed $v$ relative to the observer-source line of sight,

$$
t_{\mathrm{E}}=\frac{r_{\mathrm{E}}}{v}, \quad r_{\mathrm{E}}^{2}=\frac{4 G M d_{\mathrm{ol}} d_{\mathrm{ls}}}{c^{2} d_{\mathrm{os}}} .
$$

Here $r_{\mathrm{E}}$ is the Einstein radius and $d_{\mathrm{ol}}, d_{\mathrm{ls}}$, and $d_{\mathrm{os}}$, are the distances between the observer, lens, and source. Hence, for most events one cannot tell how far the lens is or how fast it is going, characteristics that, if known, would clearly distinguish between the halo-lens and LMC-lens hypotheses.

\footnotetext{
${ }^{1}$ Alfred P. Sloan Foundation Fellow.
}

The low overall event detection rate exacerbates the problem of lack of information about individual events. For a small fraction of events it is possible to extract additional information. For example, if the lens is a binary and the source crosses the caustic in the binary-lens magnification pattern, then one can measure the time it takes for the lens to cross the source angular radius (known from its color, flux, and the Planck law) and so determine the proper motion of the lens. In fact, this was how one of the two SMC events was inferred to be self-lensing. If the event is sufficiently long, the reflex motion of the Earth gives rise to parallax distortions of the light curve (Gould 1992), and if the source is bright enough to allow measurement of this subtle effect, then one can measure the combination $\tilde{v}=$ $\left(d_{\mathrm{os}} / d_{1 \mathrm{~s}}\right) v$, which is very different for LMC and halo lenses. This is how the other SMC event was inferred to be selflensing. Another rare (and not yet definitively observed) effect that can yield a proper motion measurement is a binary source (Han \& Gould 1997). In the future, it may be possible to measure parallaxes and/or proper motions using the Space Interferometry Mission (Boden, Shao, \& Van Buren 1998) or the Space Infrared Telescope Facility (Gould $1999)$, but only for sufficiently bright $(V \lesssim 20)$ and hence relatively rare sources. If the detection rate could be improved, the total number of events for which more information could be extracted would likewise increase.

It is reasonable to suppose that given larger telescopes, larger and more efficient detectors, smaller point-spread functions (PSFs), fainter sky, and better weather, it would be possible to increase the event detection rate. But by how much? Would a massive series of upgrades be worth the effort and expense? After 7 years of microlensing experiments, there are no published works that address this question. It is not even known, for example, whether it is better to spend the telescope resources presently available intensively observing the brighter regions of the LMC where there are more sources, or observing these less intensively and applying the telescope time so saved to the outer regions of the LMC with lower surface brightness. 
One reason for the slow progress on this front is that the problem of estimating the detection rate for a given set of observational parameters (often called the "efficiency") is very time consuming. For example, after many years of effort, the MACHO collaboration has only recently succeeded in developing a pipeline that takes an arbitrary series of observations and returns an efficiency estimate (K. Griest 1998, private communication). To actually apply this algorithm to the accumulated data set will require many months of computer time. Hence, the determination of efficiencies for a multiplicity of hypothetical observing programs seems like an intractable problem.

Estimating the real efficiencies is complicated because the real detection algorithms are complicated. These require the formation of a template image and the identification on the template of a set of "stars." The number of such "stars" is limited by the number of resolution elements in the template image, but each "star" may be composed of several real stars whose light is all blended together. Whether lensing of one of these stars is detectable depends on the combination of other stars in and near the resolution element as well as on the temporal pattern and intensity of the observations.

However, for purposes of understanding the relative efficiency of different observational strategies, these details of the detection algorithm are not important: their effects approximately cancel when one compares one strategy with another. Moreover, present PSF-fitting detection algorithms are likely to be replaced in the future by pixel lensing (image subtraction) techniques. In contrast to PSF fitting, the mathematical description of pixel lensing is extremely simple (Gould 1996). Hence, by using the pixel-lensing formalism one can understand the whole range of possible observation strategies in terms of a few easily understood parameters. Although the absolute number of events detected by current algorithms will be overestimated by the pixel-lensing formalism, this overestimate is not likely to be more than a factor of 2 . See $\S 5.2$. More importantly, the relative number for different strategies should be quite accurate.

\section{PIXEL-LENSING FORMALISM}

In the standard microlensing formalism, one imagines that one is monitoring an isolated star of unmagnified flux $F_{0}$ and that it is magnified by a lens to a flux (Paczyński 1986)

$$
\begin{aligned}
F\left(t ; t_{0}, \beta, t_{\mathrm{E}}, F_{0}\right) & =F_{0} A\left[u\left(t ; t_{0}, \beta, t_{\mathrm{E}}\right)\right], \\
u(t) & =\left[\frac{\left(t-t_{0}\right)^{2}}{t_{E}^{2}}+\beta^{2}\right]^{1 / 2},
\end{aligned}
$$

where $t_{0}$ is the time of maximum magnification, $\beta$ is the impact parameter in units of $r_{\mathrm{E}}$, and $A(u)$ is the magnification,

$$
A(u)=\frac{u^{2}+2}{u\left(u^{2}+4\right)^{1 / 2}} .
$$

Actually, in crowded fields one can never assume that the source star is truly isolated. In fact, even isolated stars can have luminous binary companions, or the lens could be luminous. Hence one must generally write equation (2) as $F\left(t ; t_{0}, \beta, t_{\mathrm{E}}, F_{0}, B\right)=F_{0} A[u(t)]+B$, where $B$ is the sum total of all unlensed sources in the aperture. This can in turn be rewritten,

$$
F\left(t ; t_{0}, \beta, t_{\mathrm{E}}, F_{0}, \widetilde{B}\right)=F_{0}\left\{A\left[u\left(t ; t_{0}, \beta, t_{\mathrm{E}}\right)\right]-1\right\}+\widetilde{B},
$$

where $\widetilde{B}=F_{0}+B$ is the baseline flux. Since the baseline flux is ordinarily well measured by the numerous observations away from the event, it can easily be subtracted from the remaining flux measurements. Hence, equation (4) can effectively be rewritten,

$$
\tilde{F}(t)=F(t)-\widetilde{B}=F_{0}\{A[u(t)]-1\} .
$$

Equation (5) was originally written to describe lensing toward M31, not the LMC. For M31, one does not begin with the delusion that one is monitoring an isolated star because the field contains virtually no resolved stars. Rather, one recognizes that the only observable quantity is the difference in flux $\widetilde{F}(t)$ between the present epoch and the baseline (Crotts 1992; Baillon et al. 1993). Consider a single observation with exposure time $t_{\exp }$ by a telescope that records $\alpha$ electrons per unit flux per unit time. Then the signal-to-noise ratio, $Q$, of the observation is

$$
Q(t)=\frac{F_{0}\{A[u(t)]-1\} \alpha t_{\text {exp }}}{\left[\left\{S_{t} \Omega+F_{0} A[u(t)]\right\} \alpha t_{\text {exp }}\right]^{1 / 2}} .
$$

where $\Omega_{\mathrm{psf}}$ is the angular area of the PSF and $S_{t} \Omega_{\mathrm{psf}}$ is the total flux (including neighboring stars plus sky) inside the aperture. For M31, the surface brightness is sufficiently uniform that $S_{t}$ can be taken to be the average surface brightness near the source. For the LMC, this approximation no longer holds: sometimes the galaxy light falling into the aperture will be significantly more than average and sometimes less. I will assume that for the purpose of estimating efficiencies, these variations cancel out, and I adopt equation (6) as written. Suppose that a series of observations are made roughly uniformly over the event, between times $t_{-}$and $t_{+}$, with a mean exposure time per day $t_{\text {exp }}$, always with the same seeing. Then $\Delta \chi^{2}$, the square of the total signal-to-noise ratio is given by

$$
\Delta \chi^{2}=\sum_{i} Q^{2}\left(t_{i}\right)=\alpha t_{\exp } \frac{t_{e}}{\text { day }} G\left(S_{t} \Omega_{\mathrm{psf}}, F_{0}, \beta, \tau_{ \pm}\right),
$$

where,

$$
G\left(F_{s}, F_{0}, \beta, \tau_{ \pm}\right)=\int_{\tau_{-}}^{\tau_{+}} d \tau \frac{F_{0}^{2}\{A[u(\tau, \beta)]-1\}^{2}}{F_{s}+F_{0} A[u(\tau, \beta)]},
$$

and where $\tau \equiv\left(t-t_{0}\right) / t_{\mathrm{E}}$, and $F_{s} \equiv S_{t} \Omega_{\mathrm{psf}}$. For $\left|\tau_{ \pm}\right| \gtrsim 1.5$, $\mathrm{G}$ is only weakly dependent on $\tau_{ \pm}$. For simplicity, I will henceforth adopt $\tau_{ \pm}= \pm 2$ and remove $\tau_{ \pm}$as arguments of $G$.

\section{LUMINOSITY FUNCTION}

I construct a luminosity function (LF) from the observed apparent $R$ band LF of the MACHO collaboration (D. Alves 1998, private communication) and the absolute $M_{V}$ band LF of Holtzman et al. (1997) derived from Hubble Space Telescope (HST) data. For the latter, I first recover the observed $V$ band LF by adding the distance modulus of the LMC $\left(\mu_{\mathrm{LMC}}=18.5\right)$ and the extinction $[E(B-V)=0.1]$ adopted by the authors. I then convert to $R$ band using the relation $V-R=\left(M_{V}-2.89\right) / 6.74$. This is actually valid only for main-sequence stars, but these are the vast majority 


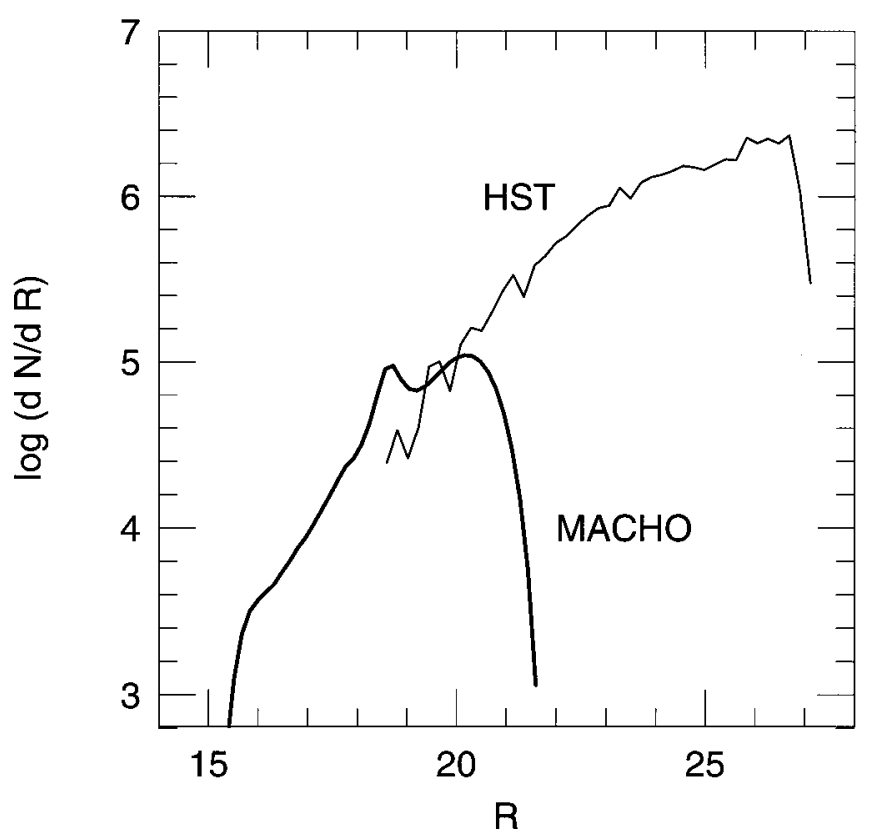

FIG. 1.-Luminosity functions (LFs) for the LMC normalized so that the integrated flux is $R=3.85$, i.e., 10 de Vaucouleurs (1957) surfacebrightness units integrated over $1 \mathrm{deg}^{2}$. The complete LF is constructed from the MACHO LF (bold) (D. Alves 1998, private communication) for $R \leq 20$ and the HST LF (solid) (Holtzman et al. 1997) for $R>20$. The $H S T$ LF is first transformed from $V$ to $R$ band before being plotted here. The relative normalization between the two LFs is set from the overlap region $19<R<20$.

of the HST stars, and in any event the $V$-to- $R$ conversion has almost no impact on the results. The two LFs are shown in Figure 1. The MACHO data become incomplete for $R \gtrsim 20$. The HST data suffer from small number statistics for $R \lesssim 19$. I therefore match the two by eye in the overlap region (as indicated in Fig. 1) and construct the final LF by using MACHO for $R \leq 20$ and $H S T$ for $R>20$. Note that the HST LF itself suffers from serious incompleteness for $R>26$. However, this has almost no impact on the present study since these fainter stars contribute very little to the total light (and so to the normalization of the LF) and even less to observable microlensing events. The LF in Figure 1 is normalized to a total flux $F_{*}$ corresponding to $R=3.85$. This is the integrated light in $1 \mathrm{deg}^{2}$ assuming 10 times the unit surface brightness arbitrarily adopted by de Vaucouleurs (1957) for his surface brightness map of the LMC. A region with $F_{*} \mathrm{deg}^{-2}$ has a surface brightness of $R=21.63$, which is typical of the inner 10 $\mathrm{deg}^{2}$ of the LMC. I will therefore use this unit of integrated flux throughout this paper,

$$
\frac{F}{F_{*}}=10^{-0.4(R-3.85)} \text {. }
$$

\section{EVENT DETECTION FUNCTIONS}

I now suppose that all events with $\Delta \chi^{2}$ greater than some minimum $\Delta \chi_{\min }^{2}$ are detected. For each star of flux $F_{0}$, and impact parameter $\beta$, one can therefore define a minimum exposure time (per day) required for detection of the event (see eq. [7])

$$
t_{\exp }=\frac{\Delta \chi_{\min }^{2}}{\alpha\left(t_{\mathrm{E}} / \text { day }\right) G\left(F_{s}, F_{0}, \beta\right)}
$$

I now assume that $\Delta \chi_{\min }^{2}, \alpha$ and $t_{\mathrm{E}}$ are all fixed. For $\Delta \chi_{\min }^{2}, \mathrm{I}$ adopt the value used by the MACHO collaboration in their $2 \mathrm{yr}$ LMC study, $\Delta \chi_{\min }^{2}=500$ (Alcock et al. 1997a). I adopt $t_{\mathrm{E}}=40$ days, the typical timescale measured by MACHO (Alcock et al. 1997a). Of course, the actual observed values of $t_{\mathrm{E}}$ cover a broad range of a factor $\sim 8$. However, I show below that this simplifying assumption has almost no impact on the results. I adopt $\alpha=125 \mathrm{~s}^{-1}$ at $R=20$, corresponding to what is expected from a $2.5 \mathrm{~m}$ telescope with a thinned CCD and standard Cousins $R$ filter. These are the characteristics of the "next generation" microlensing experiment proposed by Stubbs (1998). I will consistently use the "next generation" characteristics in my initial example. After fixing these parameters, $t_{\exp }$ is a function only of $F_{s}, F_{0}$, and $\beta$. I then integrate over the LF and a uniform distribution in $\beta$ to obtain the event rate as a function of the minimum daily exposure time necessary to observe them,

$$
\begin{aligned}
\frac{d \Gamma_{i}}{d t_{\exp }}= & \frac{2}{\pi} \frac{\tau}{t_{\mathrm{E}}} \frac{S_{i} \Omega_{\mathrm{ccd}}}{F_{*}} \int_{0}^{0.66} d \beta \int d F_{0} \Phi\left(F_{0}\right) \\
& \times \delta\left[t_{\exp }-\frac{\Delta \chi_{\min }^{2}}{\alpha\left(t_{\mathrm{E}} / \mathrm{day}\right) G\left(F_{s}, F_{0}, \beta\right)}\right],
\end{aligned}
$$

where $\delta$ is the Dirac $\delta$-function, $\Phi$ is the LF normalized to $F_{*}$ (see Fig. 1), $S_{i}$ is the surface brightness of field $i$, and $\Omega_{\text {ccd }}$ is the area of the CCD. I assume an optical depth $\tau=2.9 \times 10^{-7}$, the best-fit value for the MACHO $2 \mathrm{yr}$ study (Alcock et al. 1997a). Note that I have restricted the integration to $\beta \leq 0.66$, corresponding to a minimum peak magnification $A_{\text {peak }} \geq 1.75$, again following the MACHO selection criteria (Alcock et al. 1997a). Figure 2 shows the normalized cumulative distribution function

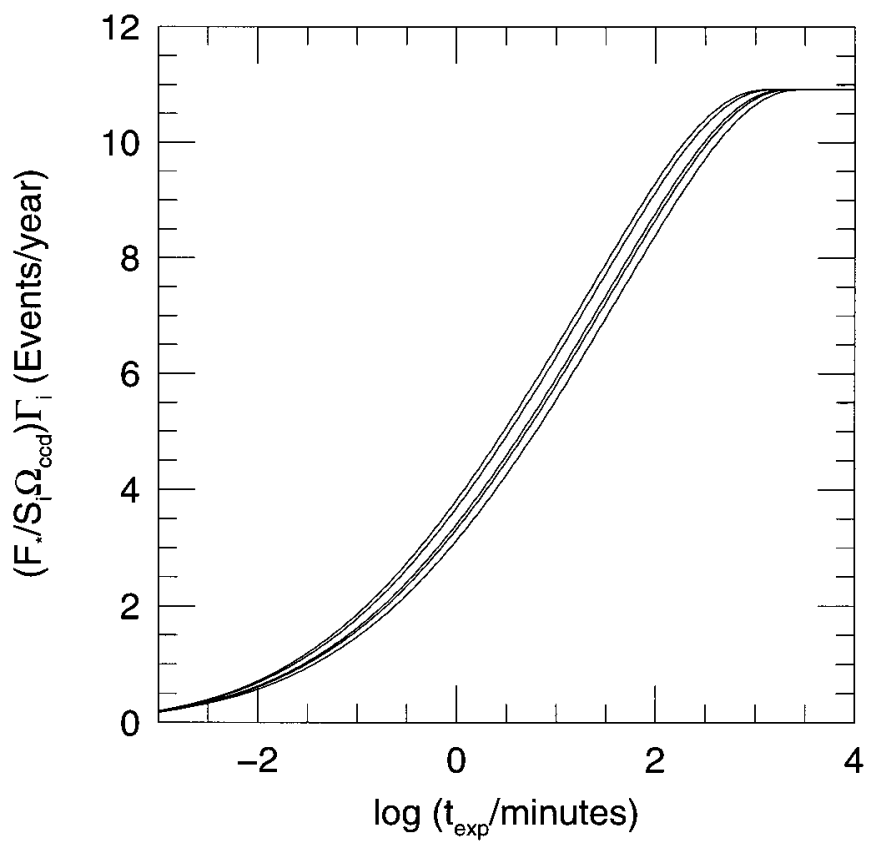

Fig. 2.-Event rate per year per $F_{*}$ of LMC flux, as a function of exposure time per day. Here $F_{*}$ (eq. [9]) is the flux corresponding to $R=3.85$ (which is typical of the flux from $1 \mathrm{deg}^{2}$ in the central $10 \mathrm{deg}^{2}$ of the LMC). To obtain the true rate, multiply by $S_{i} \Omega_{\mathrm{ccd}} / F_{*}$. Shown (left to right) are curves for surface brightness $0.12,0.41,1.2,1.5$, and $2.3 F_{\text {* }} \mathrm{deg}^{-2}$, corresponding to a range of $R=23.93$ to $R=20.73 \mathrm{mag} \mathrm{arcsec}^{-\frac{*}{2}}$. Characteristics of the "next generation" microlensing experiment have been assumed: $2.5 \mathrm{~m}$ telescope with thinned CCDs, sky brightness of $R=21.0$ $\operatorname{mag} \operatorname{arcsec}^{-2}$, PSF size $\Omega_{\mathrm{psf}}=\pi \operatorname{arcsec}^{2}$. 
$\left(F_{*} / S_{i} \Omega_{\mathrm{ccd}}\right) \Gamma_{i}\left(t_{\mathrm{exp}}\right)$ where $\Gamma\left(t_{\mathrm{exp}}\right)$ is the integral of equation (11), assuming a 180-day observing season per year. Five different values of LMC surface brightness are shown ranging from $S_{\mathrm{LMC}}=2.3 F_{*} \mathrm{deg}^{-2} \quad(R=20.73 \mathrm{mag}$ $\operatorname{arcsec}^{-2}$ ) characteristic of the LMC bar to $S_{\mathrm{LMC}}=0.12 F_{*}$ $\operatorname{deg}^{-2}\left(R=23.93 \mathrm{mag} \operatorname{arcsec}^{-2}\right)$ characteristic of the region $\sim 5^{\circ}$ from the LMC center. For each of these calculations, I have assumed a sky $S_{\text {sky }}$ of $R=21.0 \mathrm{mag} \mathrm{arcsec}^{-2}$, and a PSF size $\Omega_{\mathrm{psf}}=\pi \operatorname{arcsec}^{2}$.

Most of the conclusions of this paper can be extracted from a careful inspection of Figure 2. First, the five curves look very similar, differing by only $\sim 18 \%$ at the canonical exposure time $t_{\exp }=5$ minutes. This means that, for fixed exposure time, the number of detectable events is essentially proportional to the surface brightness (which has been factored out of Fig. 2). Second, the slope of these curves at $t_{\exp }=5$ minutes is $d \ln \Gamma / d \ln t_{\exp } \sim 0.23$. That is, a factor 2 increase in exposure time increases the rate of event detection by only $\sim 16 \%$. Hence, faced with the choice of doubling the exposure time on a high surface-brightness field or observing a new field with $1 / 5$ the surface brightness, one should choose the latter. In fact, I will show in $\S 5$, that essentially the whole LMC should be monitored. Third, the event rate for the canonical $t_{\exp }=5$ minute exposures is surprisingly high, $\sim 5 F_{*}^{-1}$ events per $\mathrm{yr}^{-1}$. Since the total flux from the LMC is $\sim 36 F_{*}$, this implies that over 100 events $\mathrm{yr}^{-1}$ could be detected if the survey covered the whole LMC.

Figure 2 also allows one to understand why using the average event timescale is adequate for predicting the total event rate. From equation (7), it follows that events that are a factor of two shorter than average suffer the same loss of signal-to-noise ratio as events with half the exposure time. Hence, they suffer the same loss of detection rate, i.e., $23 \%$. This means that over the entire factor $\sim 8$ range of observed timescales, there is only a few tens of percent difference in detection rate. Thus, the detection rate for the mean timescale is an excellent proxy for the mean detection rate.

At this point, it is appropriate to estimate the effect of some of the other simplifying assumptions of this analysis. First, I have assumed that three time-variable quantities could be replaced by their time-averaged values, namely, the seeing, the sky brightness, and the time lost to weather and the Moon. These assumptions are all strictly valid in a mathematical sense in the limit where the characteristic timescale of the fluctuations is short compared to the event timescale. In this case, the values of $A(u)$ entering $Q$ in equation (6) are all very similar during the temporal fluctuation, so all that is important is the average values of the quantities on the right-hand side of this equation. (More exactly, what is important is the average value of the square of the entire expression. For example, for sources below the sky, it is not the average seeing, but the mean inverse square of the seeing that enters the total signal-to-noise ratio. Thus a few nights of 8 " seeing do not radically degrade the "average seeing": they just count as lost nights.) Since, the typical event timescale is 40 days, while the timescales for weather patterns and major interruptions from the lunar cycle are typically a week, the use of average values is well justified. However, events have been detected that are as short as 2 weeks, and for these the assumptions begin to break down. In addition, for very low impact-parameter events, the effective timescale is much shorter than the Einstein crossing time, so the assumptions also break down for these events.
A similar analysis can be made for the assumption that surface brightness fluctuations can be ignored, again using equation (6). What enters the signal-to-noise ratio is the inverse of the surface brightness. As with the seeing, a few bright spots near bright stars do not significantly degrade the average condition. For regions of modest variation, the same argument given above (using Fig. 2) that one can use the average timescale also implies that one can use the average surface brightness.

\section{OPTIMAL STRATEGIES}

The formalism developed in the previous section can be used to estimate the event detection rate for various observational programs and to optimize detection efficiency for a given set of equipment. I first analyze the " next generation" experiment (whose characteristics are reflected in Fig. 2) and then compare this with the current MACHO (Alcock et al. 1997a) and EROS (Ansari et al. 1997) experiments.

\subsection{Next Generation}

As described in $\S 4$, the "next generation" experiment proposed by Stubbs (1998) would have a $2.5 \mathrm{~m}$ telescope, an $\Omega_{\text {ccd }}=1 \mathrm{deg}^{2}$ camera with thinned CCDs (and so $\alpha=125$ $e^{-} \mathrm{s}^{-1}$ at $\left.R=20\right)$, a dark sky $(R=21.0 \quad \mathrm{mag}$ $\left.\operatorname{arcsec}^{-2}\right)$, and a small PSF $\left(\Omega_{\mathrm{psf}}=\pi \operatorname{arcsec}^{2}\right)$.

Let $\Gamma_{i}\left(S_{i}, S_{\text {sky }}, t_{\text {exp }}\right)$ be the event rate for a $1 \mathrm{deg}^{2}$ field with surface brightness $S_{i}$, and background flux $F_{s}=$ $\Omega_{\mathrm{psf}}\left(S_{i}+S_{\text {sky }}\right)$. The total rate of detectable events is then

$$
\Gamma_{\mathrm{tot}}=\sum_{i} \Gamma_{i}\left(S_{i}, S_{\mathrm{sky}}, t_{\mathrm{exp}}\right) \text {. }
$$

I maximize $\Gamma_{\text {tot }}$ subject to a constraint on the total amount of observing time. I assume an average of $6.5 \mathrm{hr}$ per night are available for observations over a 180 day observing season and that 49 minutes of this time are lost to overhead (readout and pointing). I discuss this figure further below. I assume that $20 \%$ of the time is lost to weather and $25 \%$ of the remaining time is lost to (or at any rate degraded by) the Moon. I construct an $11^{\circ} \times 11^{\circ}$ surface-brightness grid using the de Vaucouleurs (1957) map. I find a total event rate $\Gamma_{\text {tot }}=129 \mathrm{yr}^{-1}$, with a distribution of exposure times shown in Figure 3. Note that the exposure times are roughly proportional to surface brightness. This can be understood from Figure 2: if the curves were exactly straight lines and were completely independent of surface brightness, then the proportionality would be exact. That is, we would have $\Gamma_{i}=C_{1} S_{i} \ln \left(C_{2} t_{\exp }\right)$, so that $d \Gamma_{i} / d t_{\exp }=$ $C_{1} S_{i} / t_{\text {exp }}$ where $C_{1}$ and $C_{2}$ are constants. Detection is maximized when these derivatives are equal in all fields, which occurs if $t_{\exp } \propto S_{i}$. The inner fields are typically observed for about 5 minutes, while the outer fields are typically observed for 1 minute or less. I assume that there is 1 minute of telescope overhead time per exposure, so these very short exposures in the outer fields seem wasteful. I therefore assume that the inner $25 \mathrm{deg}^{2}$ are observed every available night while the outer $96 \mathrm{deg}^{2}$ are observed only every third night. This schedule accounts for my estimate of 49 minutes of overhead per night.

The total event rate is actually not very sensitive to the exact observation strategy, provided that the whole LMC is observed. For the optimal exposure time, there are 129.0 events $\mathrm{yr}^{-1}, 69.5$ in the inner $25 \mathrm{deg}^{2}$ and 59.5 in the outer $96 \mathrm{deg}^{2}$. However, if exposure times are set to be equal, I find 127.3 events with 67.3 in the inner fields and 60.0 in the 


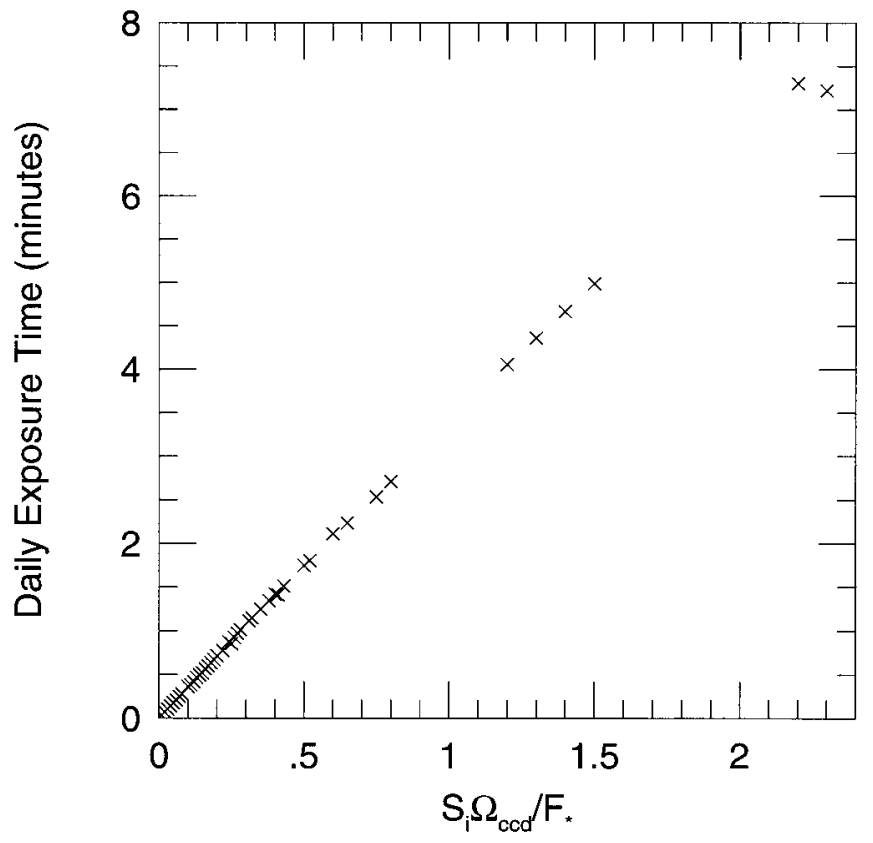

FIG. 3.-Optimal distribution of exposure times for 121 LMC fields of 1 $\mathrm{deg}^{2}$. Exposure times are chosen to maximize the total number of events assuming uniform optical depth across the LMC. The abscissa is the surface brightness $S$ in units of $R=21.63 \mathrm{mag}$ arcsec. Assumptions are the same as in Fig. 2. The optimal exposure time is almost exactly proportional to $S$. However, equal exposure times in all fields reduces the total number of events by only about $2 \%$.

outer fields. On the other hand, if only the inner $25 \mathrm{deg}^{2}$ is observed (and the overhead time is consequently cut to 25 minutes), then only 70.9 events are expected. This confirms the conclusion I drew from inspection of Figure 2 that additional telescope time is better spent on low-surfacebrightness fields than on intensive monitoring of the inner fields.

Clearly, however, when the surface brightness falls sufficiently low, it must be counterproductive to observe a field. To determine that point, I return to the optimal solution. As noted above, $d \Gamma_{i} / d t_{\exp }$ must be equal in all fields, and its value is 0.18 events $^{-1} \mathrm{yr}^{-1}$ minute ${ }^{-1}$. Since, the overhead for the outer fields is $1 / 3$ minute, this implies that observation of a field is counterproductive if the event rate falls below 0.06 events $\mathrm{yr}^{-1}$. I find that this occurs at $S \Omega_{\mathrm{ccd}}=$ $0.02 F_{*}\left(R=25.9 \mathrm{mag} \operatorname{arcsec}^{-2}\right)$, which is generally fainter than the inner $121 \mathrm{deg}^{2}$.

\subsection{MACHO Experiment}

I now apply the same formalism to the MACHO experiment (Alcock et al. 1997a). I assume $\alpha=31 e^{-} \mathrm{s}^{-1}$ at $R=20$ corresponding to a $1.25 \mathrm{~m}$ telescope with unthinned CCDs but a broader $R$ passband. I assume a brighter sky $\left(R=19.5 \mathrm{mag} \operatorname{arcsec}^{-2}\right)$, a larger PSF $\left(\Omega_{\mathrm{psf}}=4 \pi \operatorname{arcsec}^{2}\right)$, and a smaller camera $\Omega_{\text {ccd }}=0.5 \mathrm{deg}^{2}$. I assume a $50 \%$ time loss to weather, but only $15 \%$ to the Moon (because the sky is already so bright). I continue to assume 1 minute of over- head per exposure. I then find a total of 27.6 events $\mathrm{yr}^{-1}$, or 15.8 if observations are restricted to the inner $25 \mathrm{deg}^{2}$.

As a consistency check, it is important to try to make contact with the 2 yr MACHO results based on an inner region of $11 \mathrm{deg}^{2}$. Eight candidate events were detected. Recall that I normalized the event rate to the optical depth $\left(\tau=2.9 \times 10^{-7}\right)$ estimated by MACHO based on these eight events. Since MACHO spent substantial time observing other regions of the LMC (even though they only reported on these $11 \mathrm{deg}^{2}$ ), I mimic the MACHO observations by assuming that the inner $25 \mathrm{deg}^{2}$ were monitored, but count events only for the brightest $11 \mathrm{deg}^{2}$. I then find 10.6 events $\mathrm{yr}^{-1}$, substantially more than the four events per year actually observed.

Part of the difference is undoubtedly due to the fact that I have assumed a pixel-lensing analysis, while MACHO carried out a Dophot-based analysis. Any unresolved stars that happened to lie within the PSF of a template "star" will be effectively monitored and so subject to detection in a Dophot-based analysis. However, lensing of unresolved stars lying between template "stars" will be missed. Melchior et al. (1998) also concluded that a pixel-lensing analysis of LMC observations would increase the event detection rate substantially. On the other hand, it is possible that part of the difference between the 10.6 events predicted in my analysis and the four observed by MACHO is that my simplified analysis fails to reflect real effects that would diminish the effectiveness of both a Dophot-based and a pixel-lensing analysis. (Note, however, that Poisson statistics is not a possible cause since my analysis was normalized to the optical depth based on the four events actually detected.) To the extent that the difference between the 10.6 events predicted and 4 events observed is due factors that are common to a pixel-lensing and Dophot-based analyses, my estimates of the event rate in a "next generation" experiment should also be scaled down.

\subsection{EROS Experiment}

For the EROS II experiment, I assume $\alpha=20 e^{-} \mathrm{s}^{-1}$ at $R=20$ corresponding to a $1 \mathrm{~m}$ telescope with unthinned CCDs, $\Omega_{\mathrm{psf}}=4 \pi \operatorname{arcsec}^{2}$, and a camera size $\Omega_{\mathrm{ccd}}=1 \mathrm{deg}^{2}$. I assume sky, weather, and Moon conditions similar to the "next generation" parameters, and telescope overhead of 2.5 minutes. I find a total of 46.1 events year $^{-1}$, of which 24.7 are in the inner $25 \mathrm{deg}^{2}$, or a total of 25.5 if observations are restricted to the inner $25 \mathrm{deg}^{2}$. EROS carries out a number of nonmicrolensing projects that reduce the time available for LMC observations, so these rates may be slightly overestimated.

I thank C. Stubbs for his persistent prodding, A. Becker for help determining the LMC luminosity function, and B.S. Gaudi for a careful reading of the manuscript. This work was supported in part by grant AST 97-27520 from the NSF.

\section{REFERENCES}

Afonso, C., et al. 1998, A\&A, 337, L17

Albrow, M., et al. 1999, ApJ, 512, 000

Alcock, C., et al. 1997a, ApJ, 486, 697 1997b, ApJ, 491, L11 1999, ApJ, 518, in press

Ansari, R., et al. 1997, A\&A, 324, L69
Baillon, P., Bouquet, A., Giraud-Héraud, Y., \& Kaplan, J. 1993, A\&A, 277, 1

Boden, A. F., Shao, M., \& Van Buren, D. 1998 ApJ, 502, 538

Crotts, A. P. S. 1992, ApJ, 399, L43

de Vaucouleurs, G. 1957, AJ, 62, 69

Gould, A. 1992, ApJ, 392, 442 


\section{GOULD}

Gould, A. 1996, ApJ, 470, 201

1999, ApJ, 514, 869

Han, C., \& Gould, A. 1997, ApJ, 480, 196

Holtzman, J. A., et al. 1997, AJ, 113, 656

Palanque-Delabrouille, N., et al. 1998, A\&A, 332, 1

Melchior, A.,-L., et al. 1998, A\&A, 134, 377
Paczyński, B. 1986, ApJ, 304, 1

Rhie, S. H., Becker, A. C., Bennett, D. P., Fragile, P. C., Johnson, B. R., King, L. J., Peterson, B. A., \& Quinn., J. 1999, ApJ, submitted

Stubbs, C. 1998, preprint (astro-ph/9810488)

Udalski, A., Szymański, M., Kubiak, M., Pietrzyński, G., Woźniak, P., \& Żebruń, K. 1998, Acta Astron., 48, 147 